\title{
Proof-of-Principle of High-Fidelity Coupled CRUD Deposition and Cycle Depletion Simulation
}

\author{
Daniel J. Walter, Victor Petrov, Annalisa Manera, Benjamin Collins, Thomas \\ Downar \\ Department of Nuclear Engineering and Radiological Sciences, University of Michigan, \\ 2355 Bonisteel Boulevard, Ann Arbor, 48109-2104 Michigan, USA, \\ Brian K. Kendrick \\ Theoretical Division (T-1, MS B221) Los Alamos National Laboratory Los Alamos, New \\ Mexico 87545, USA,
}

\begin{abstract}
A multiphysics framework for the high-fidelity simulation of CRUD deposition is developed to better understand the coupled physics and their respective feedback mechanisms. This framework includes the primary physics of lattice depletion, computational fluid dynamics, and CRUD chemistry. The three physics are coupled together via the operator-splitting technique, where predictor-corrector and fixed-point iteration schemes are utilized to converge the nonlinear solution. High-fidelity simulations may provide a means to predict and assess potential operating issues, including CRUD induced power shift and CRUD induced localized corrosion, known as CIPS and CILC, respectively. As a proof-of-principle, a coupled 500-day cycle depletion simulation of a pressurized water reactor fuel pin cell was performed using the coupled code suite; a burnup of $31 \mathrm{MWd} / \mathrm{kgHM}$ was reached. The simulation recreated the classic striped CRUD pattern often seen on pulled fuel rods containing CRUD. It is concluded that the striping is caused by the flow swirl induced by spacer grid mixing vanes. Two anticorrelated effects contribute to the striping: (1) the flow swirl yields significant azimuthal temperature variations, which impact the locations where CRUD deposits, and (2) the flow swirl is correlated to increased shear stress along the cladding surface and subsequent erosion of the CRUD layer. The CIPS condition of the core is concluded to be primarily controlled by lithium tetraborate precipitation, referred to as boron hideout, which occurs in regions experiencing subcooled nucleate boiling as soluble boron and lithium species reach their solubility limit within the CRUD layer. Subsequently, a localized reduction in power occurs due to the high neutron absorption cross section of boron-10.
\end{abstract}

Email addresses: djwalter@umich.edu (Daniel J. Walter), petrov@umich.edu (Victor Petrov), manera@umich.edu (Annalisa Manera), bscollin@umich.edu (Benjamin Collins), downar@umich.edu (Thomas Downar), bkendric@lanl.gov (Brian K. Kendrick)

Preprint submitted to Elsevier

April 27, 2015 
Keywords: CRUD, CIPS, axial offset, high-fidelity, multiphysics, CILC

\section{Introduction}

Optimal PWR performance relies on the consideration of a variety of design parameters, risk assessments, constraints, and trade-offs. Chalk River unidentified deposit (CRUD) originally surfaced in the late 1940s at the Canadian Chalk River Laboratories. Subsequently, two primary operating concerns were identified. CRUD induced power shift (CIPS), historically known as axial offset (AO), refers to the shifting of the axial power distribution, often toward the bottom half of the core. This is due to preferential CRUD build-up in the top half of the core due to higher coolant temperature and increased probability of subcooled nucleate boiling (SNB) [8]. CRUD induced localized corrosion (CILC) refers to the corrosion of the cladding due to increased thermal insulation of the cladding surface by the CRUD layer; this is illustrated in Figure 1. The risk of CIPS and CILC increases with power uprates and longer cycles due to the increased potential for thicker CRUD deposits. Therefore, CIPS and CILC are important phenomena that require careful risk assessment because they impact core peaking factors, shutdown margin, and fuel integrity.

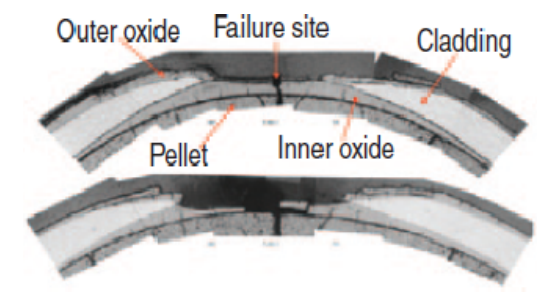

Figure 1: CRUD induced localized corrosion of fuel cladding [7].

Accurate modeling of the physics involved in CRUD deposition requires the use of high-fidelity, multiphysics simulations. The single-phase fluid flow field is calculated using CFD, and the internal boiling within the CRUD layer is calculated using the CRUD chemistry code. Specifically, the turbulence induced by the spacer grid mixing vanes and the locations of localized regions of higher outer cladding surface temperature, known as hot spots, must be considered for accurate CRUD predictions. Current, industry-standard, subchannel and nodal neutron diffusion codes cannot sufficiently resolve the most important physics.

The approach adopted within this computational framework involves three high-fidelity three-dimensional (3-D) coupled physics, which include lattice depletion, computational fluid dynamics (CFD), and CRUD chemistry. Due to the strong impact of the cladding surface temperature on the CRUD deposition, the CFD model includes explicit representation of the spacer grid mixing vanes, as shown in Figure 2. Two-way feedback is modeled in each of the physics interfaces, thus, accounting for the dynamic feedback processes during the depletion cycle. Coupling is achieved via the operator-splitting technique, where 


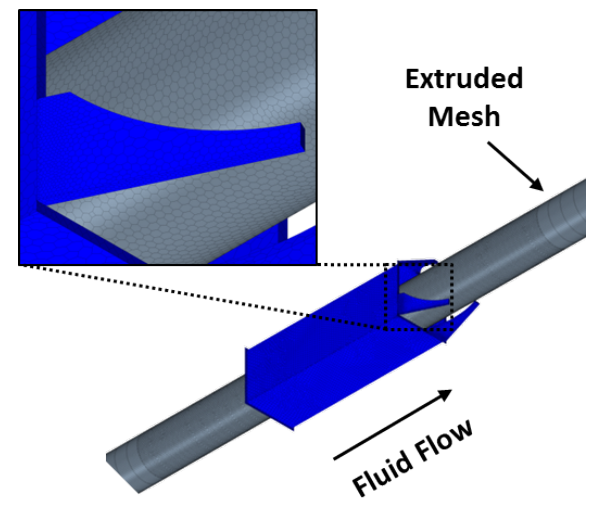

Figure 2: CFD geometry and mesh model of single pin cell with spacer grid and mixing vanes.

predictor-corrector (P-C) and fixed-point iteration (FPI) schemes are utilized to converge the nonlinear solution. However, the two time-dependent physics of nuclide depletion and CRUD deposition are not time-consistent, but are lagged in this simulation.

It is stressed that this article describes a proof-of-principle of the coupled physics of CRUD deposition and is not focused on the particular temporal coupling or solution transfer techniques. Additionally, the capability of the framework is demonstrated using the computational tools of DeCART (Deterministic Core Analysis based on Ray Tracing), STAR-CCM+, and MAMBA (MPO Advanced Model for Boron Analysis). Verification and validation of the coupled code suite has not been performed, but the expectation is that this initial demonstration contributes to the on-going effort to develop more advanced predictive CRUD capabilities.

\section{CRUD Deposition Physics}

\subsection{Corrosion Products}

In pressurized water reactors (PWRs), the primary CRUD source comes from corrosion of the steam generator tubing, which develops as oxygen diffuses into the base metal, transforming the alloy elements from the metallic state to the oxide state. Divalent metal ions are then released into the water as soluble metal ions [3]. The metal ion release rate is dependent on the diffusion coefficient of oxygen, the oxide thickness and composition, and coolant conditions, among other contributors [9]. Three coolant chemistry conditions that promote corrosion and release of the corrosion products are high oxygen content, low $\mathrm{pH}$, and high temperature. High oxygen content increases the availability of oxygen during the oxidation process. The corrosion product release rate is significantly affected by the coolant $\mathrm{pH}$. Historically, increasing the $\mathrm{pH}$ in plants has decreased corrosion; however, a high $\mathrm{pH}$ requires a high lithium concentration in 


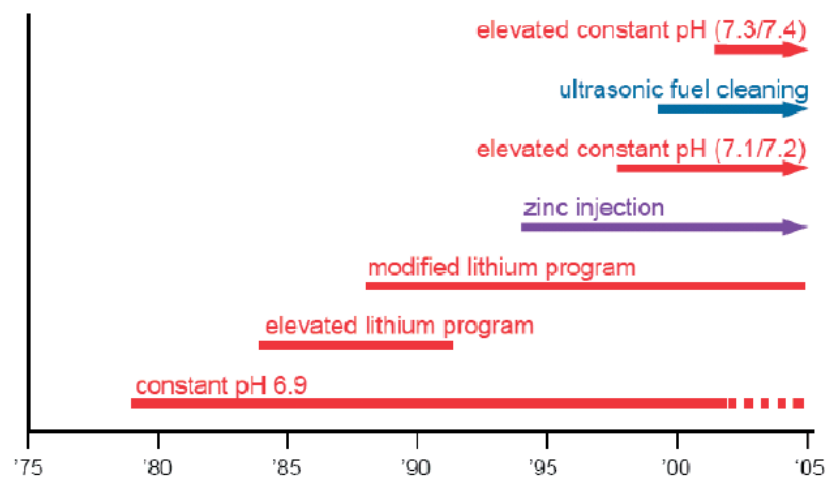

Figure 3: CRUD prevention techniques [13].

the coolant which may increase the probability of undesirable boron hideout in the CRUD layer [9]. Additional, CRUD prevention techniques developed and implemented in the last forty years are illustrated in Figure 3.

The corrosion particulates release from where they were formed, circulate the primary loop, and deposit on core internals including the fuel rods. Figure 4 shows heavy CRUD deposition on PWR fuel rods from the Palo Verde Nuclear Generating Station. These types of CRUD deposits are composed mostly of nickel ferrite, nickel oxide, and nickel metal with other nickel-iron-chrome spinels [7].

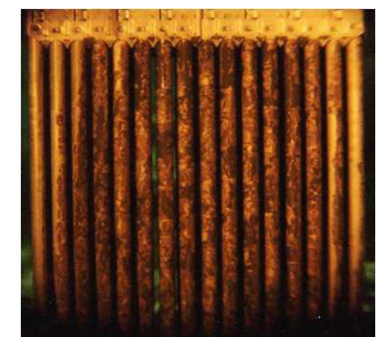

Figure 4: Heavy CRUD deposition on fuel rods at the Palo Verde Nuclear Generating Station [16].

In addition to the metallic constituents, lithium, boron and other species are often present due to Darcy flow and internal boiling within the CRUD chimneys [14]. The coolant and its soluble species, such as boric acid and lithium, are drawn into the chimneys. As the coolant vaporizes, the soluble species concentrate along the cladding surface, eventually leading to lithium tetraborate precipitation as depicted in Figure 5. The relevant reaction of precipitation of lithium tetraborate is

$$
2 \mathrm{Li}^{+}+4 \mathrm{~B}(\mathrm{OH})_{3} \rightarrow \mathrm{Li}_{2} \mathrm{~B}_{4} \mathrm{O}_{7}+5 \mathrm{H}_{2} \mathrm{O}+2 \mathrm{H}^{+} .
$$



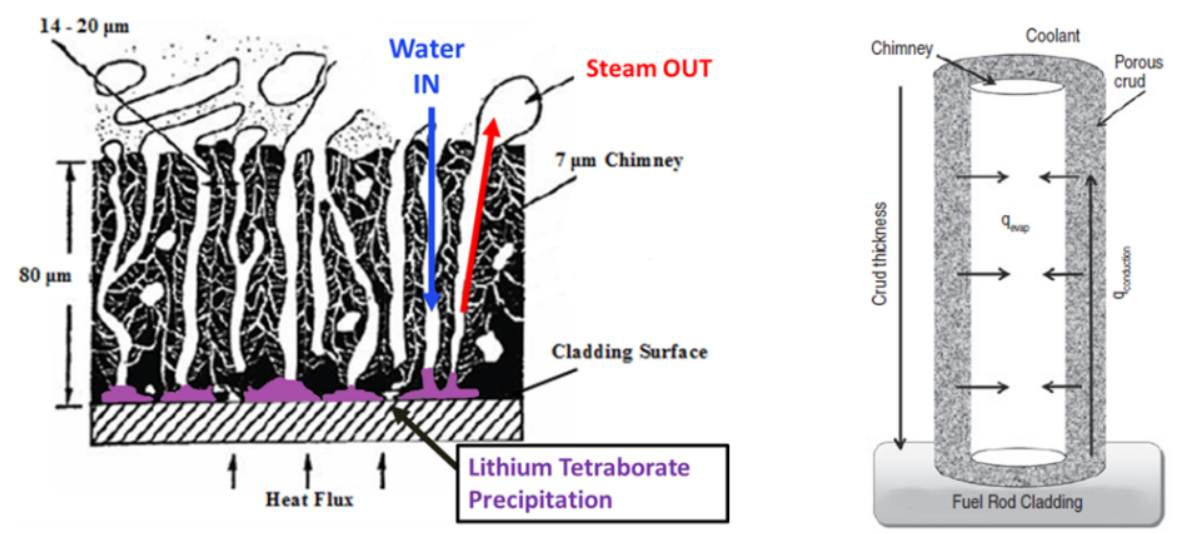

Figure 5: Sample CRUD layer showing in-flow of water and out-flow of steam in chimneys leading to preciptation (left, [14]), and the heat transfer within a chimney (right, [7]).

The highly concentrated boron residing in the thicker CRUD layers reduces the neutron flux in those regions, leading to an axial offset of the power distribution (e.g. CIPS). The thermal neutron (at an energy of $0.00253 \mathrm{eV}$ ) cross section of boron is approximately 3840 barns.

\subsection{CRUD Modeling}

In this subsection, the modeling of CRUD deposition, as implemented within the macro-scale reactor core chemistry code MAMBA, is detailed. MAMBA simulates 3-D CRUD growth along the surface of a single fuel rod. The primary physics and chemistry associated with CRUD formation currently treated in MAMBA include:

- solving a general nonlinear, 3-D heat transport equation for the CRUD layer, including localized heat sinks due to internal boiling;

- an adaptive grid which grows radially in time as mass deposits on the surface of the CRUD;

- time evolving microstructure (porosity) of the CRUD layer, due to localized deposition and precipitation of nickel ferrite and lithium tetraborate within the pores;

- time evolving lithium and boric acid coolant chemistry at the CRUD surface and inside the pores of the CRUD;

- mass transport of various soluble coolant species into the interior of the CRUD, due to boiling induced Darcy flow;

- diffusion of various soluble species inside the CRUD, due to the flow induced concentration gradients within the CRUD layer; and 
- mass evaporation in the form of steam vapor, due to the localized boiling inside the CRUD layer.

The coolant chemistry conditions are supplied by the user, or an ex-core model, and consist of the following coolant concentrations: boron $(B)$, lithium $(L i)$, dihydrogen $\left(\mathrm{H}_{2}\right)$, soluble nickel $(\mathrm{Ni})$, soluble iron $(\mathrm{Fe})$, and particulate $\mathrm{NiFe}_{2} \mathrm{O}_{4}$. The external boundary conditions needed by the 3 -D heat transport solver include the heat flux at the cladding surface and the temperature or heat transfer coefficient at the CRUD/coolant interface. The various CRUD properties used by MAMBA are based on the Westinghouse WALT loop studies [10], which include porosity, CRUD solid skeleton density and thermal conductivity, average chimney radius, and average chimney density.

The 3-D general, time-dependent, nonlinear heat equation with local heat sinks is

$$
\frac{\partial T(\vec{r}, t)}{\partial t}=\nabla \cdot\left[D_{t}(\vec{r}, t) \nabla T(\vec{r}, t)\right]-T_{\text {sink }}\left(T, T_{\text {sat }}, \eta(\vec{r})\right),
$$

where $T$ is the temperature field, $\vec{r}$ is the position vector, $t$ is the time, $T_{s a t}$ is the saturation temperature of the coolant, and $\eta$ is the CRUD porosity. The local heat sinks $T_{\text {sink }}$ are proportional to the chimney boiling by

$$
T_{\text {sink }} \propto A_{c} \kappa(\eta)\left(T-T_{\text {sat }}\right) .
$$

where $A_{c}$ is the chimney wall surface area and $\kappa$ is the permeability of the CRUD. MAMBA's boiling correlation is best described as a generalized Cohen-type thermal hydraulics model $[12,6]$. It should be clarified that the two-phase fluid models are implemented within MAMBA, while single-phase CFD calculations are performed to provide the CRUD layer boundary conditions. The thermal diffusivity $D_{t}$ is given by

$$
D_{t}(\vec{r}, T)=\frac{k(\vec{r}, T)}{C_{v}(\vec{r}, T)},
$$

where the effective thermal conductivity $k$ is calculated as a standard mixing fraction where the conductivities of water $k_{w}$ and the CRUD $k_{c}$ are weighted by the CRUD porosity,

$$
k(\vec{r}, T)=\eta(\vec{r}) k_{w}(T)+[1-\eta(\vec{r})] k_{c}
$$

The effective heat capacity is similarly determined, but is also weighted by the densities of water $\rho_{w}$ and the CRUD $\rho_{c}$,

$$
C_{v}(\vec{r}, T)=\eta(\vec{r}) \rho_{w}(T) C_{w}(T)+[1-\eta(\vec{r})] \rho_{c} C_{c} .
$$

To describe both the surface and volume deposition kinetics, the CRUD layer is deomposed into solid CRUD $c$, soluble $s$, particulate $p$, and vapor $v$ constituents. The species $i$ that are available to deposit on the surface of the CRUD are $\mathrm{NiFe}_{2} \mathrm{O}_{4}, \mathrm{NiO}$, and $\mathrm{Fe}_{3} \mathrm{O}_{4}$. The CRUD surface deposition rate 
for each species is governed by two Arrhenius rate parameters associated with the porous regions inside the CRUD layer: (i) for boiling regions $\tilde{a}_{i}^{p}$, which is multiplied by the local mass evaporation rate $q_{c}^{\prime \prime}$, i.e. the steaming flux leaving the boiling chimneys, and (ii) for non-boiling regions $a_{i}^{p}$. In boiling regions, the CRUD growth rate is enhanced due to the boiling-induced flow of coolant into the CRUD's surface, which increases the flux of particulates onto the CRUD's surface. Equation 7 gives the deposition kinetics rate equation for each surface element,

$$
\frac{d N_{i}^{c}}{d t}=a_{i}^{p} N_{i}^{p}+\tilde{a}_{i}^{p} q_{c}^{\prime \prime} N_{i}^{p}-N_{\text {erosion }}^{c},
$$

where $N_{i}^{c}$ and $N_{i}^{p}$ are the solid and particulate concentrations, respectively. The Arrhenius rate coefficient is given as

$$
a_{i}^{p}=A_{i}^{p} \exp \left[-E_{i}^{p} / R T\right],
$$

where $A_{i}^{p}$ and $E_{i}^{p}$ are the Arrhenius rate prefactor and activation energy, respectively, $R$ is the universal gas constant, and $T$ is the temperature. The CRUD loss due to turbulent flow is given as the product of the TKE $k_{C F D}$ and an adjustable constant $k_{e}$ used to correlate with plant data,

$$
N_{\text {erosion }}^{c}=k_{e} k_{C F D} .
$$

Equation 7 is solved numerically used Gear's method with automatic time step refinement [11].

The species $j$ that are available to deposit within the CRUD are $\mathrm{NiFe}_{2} \mathrm{O}_{4}$, $\mathrm{NiO}$, and $\mathrm{Fe}_{3} \mathrm{O}_{4}$, lithium tetraborate $\left(\mathrm{Li}_{2} \mathrm{~B}_{4} \mathrm{O}_{7}\right)$, lithium metaborate $\left(\mathrm{LiBO}_{2}\right)$, bonnacordite $\left(\mathrm{Ni}_{2} \mathrm{FeBO}_{5}\right)$, and metaboric acid $\left(\mathrm{HBO}_{2}\right)$. The volumetric deposition, also called pore fill kinetics, is governed by

$$
\frac{d N_{j}^{c}}{d t}=a_{j}^{s} A(\eta)\left[N_{j}^{s}-N_{j}^{*}\right],
$$

where $a_{j}^{s}$ is the Arrhenius rate, $A(\eta)$ is the pore surface area, and $N_{j}^{*}$ is the solubility of the soluble species $j$, which is a function of temperature, species concentration, and coolant $\mathrm{pH}$. The equation for CRUD porosity is

$$
\rho_{0} \frac{d \eta(\vec{r})}{d t}=-N_{j}^{c} m_{j}
$$

where $\rho_{0}$ is the nominal CRUD bulk density and $m_{j}$ is the product of the molecular mass of species $j$, concentration $N_{j}$, and volume $V_{j}$. The soluble transport equation for species $j$ is

$$
\frac{d N_{j}^{s}}{d t}=\frac{\left(F_{\text {boiling }}^{j}-F_{d i f f u s i o n}^{j}-F_{\text {vapor }}^{j}\right)}{\Delta r},
$$

where the local fluxes are defined as

$$
F_{\text {boiling }}^{j}=v_{b} N_{j}^{s}
$$




$$
F_{\text {diffusion }}^{j}=\frac{D_{j}(T) \Delta N_{j}^{s}}{\Delta L}
$$

and

$$
F_{\text {vapor }}^{j}=v_{s} N_{j}^{v},
$$

where $v_{s}$ is the steaming velocity. The steaming and boiling velocities are related through mass conservation as

$$
\rho_{s} v_{s}=\rho_{w} v_{b}
$$

The local boiling velocity is

$$
v_{b}=\frac{q_{c}^{\prime \prime \prime} \Delta r}{H \rho_{w}},
$$

$\Delta r$ is the MAMBA cell radial thickness, $\Delta L$ is the radial path length, $H$ is the effective bulk chimney heat transfer coefficient, and $q_{c}^{\prime \prime \prime}$ is the volumetric heat generation rate.

The precipitation of various solid phase boron compounds, such as $\mathrm{LiBO}_{2}$, $\mathrm{Li}_{2} \mathrm{~B}_{4} \mathrm{O}_{7}$, and $\mathrm{Ni} i_{2} \mathrm{FeBO} \mathrm{O}_{5}$ is governed by equilibrium thermodynamics and is a function of temperature. The temperature dependence of the equilibrium constants $K$ for these reactions are typically fit to experimental data using a three-term correlation of the form

$$
\log K=A+\frac{B}{T}+C \log T,
$$

where $A, B$ and $C$ are fitting constants [2]. Because lithium tetraborate is the least soluble and precipitates out of solution first (in comparison to the other boron-containing compounds of interest), this particular compound is the dominating solid boron phase within the CRUD layer modeled by MAMBA. The dominant soluble boron species is metaboric acid, which is added to the coolant within PWRs to control reactivity. The combination of the presence of lithium tetraborate and boric acid within CRUD deposits is believed to be the strongest contributor to CIPS; see the reaction given by 1 .

\section{A Multiphysics Approach}

\subsection{Governing Equations}

The governing equations associated with lattice depletion and computational

fluid dynamics are briefly introduced in this section. The primary equations of CRUD chemistry and deposition kinetics have been introduced in the previous section.

The lattice depletion physics is primarily governed by the neutron transport equation, which gives the neutron flux distribution, and the Bateman equations representing the nuclide depletion. The steady state 3-D Boltzmann transport 
equation is a function of six variables including three spatial $\vec{r}$, two angular $\vec{\Omega}$, and one energy $E$ unknown. The equation is expressed as

$$
\Omega \cdot \nabla \varphi(\vec{r}, \vec{\Omega}, E)+\Sigma(\vec{r}, E) \varphi(\vec{r}, \vec{\Omega}, E)=q_{s}(\vec{r}, \vec{\Omega}, E)+q_{f}(\vec{r}, \vec{\Omega}, E),
$$

with the source term composed of the scattering source and an assumed isotropic fission source,

$$
\begin{gathered}
q_{s}(\vec{r}, \vec{\Omega}, E)=\int_{0}^{\infty} d E^{\prime} \int_{4 \pi} d \Omega^{\prime} \Sigma_{s}\left(r, \Omega^{\prime} \rightarrow \Omega, E^{\prime} \rightarrow E\right) \varphi\left(\vec{r}, \vec{\Omega}, E^{\prime}\right) \\
q_{f}(\vec{r}, \vec{\Omega}, E)=\frac{1}{4 \pi} \frac{\chi(\vec{r}, E)}{k} \int_{0}^{\infty} d E^{\prime} \nu \Sigma_{f}\left(\vec{r}, E^{\prime}\right) \phi(\vec{r}, E) .
\end{gathered}
$$

The coefficient $\Sigma$ is the macroscopic cross section and may be decomposed into scattering $\Sigma_{s}$ and fission $\Sigma_{f}$ components as in Equations 20 and 21. The fission spectrum is represented by $\chi, k$ is the eigenvalue, and $\nu$ is the average number of neutroncs released per fission. The term multiplication factor also refers to $k$ because physically it is the asymptotic ratio of the number of neutrons in one generation and the number in the next. The angular and scalar flux are related by

$$
\phi(\vec{r}, E)=\int_{4 \pi} \varphi\left(\vec{r}, \vec{\Omega}, E^{\prime}\right) .
$$

Various assumptions and fidelity solution methods are empoyed to solve Equation 19 including the method of characteristics (MOC), discrete ordinates $\left(S_{n}\right.$ equations), and collision probability method (CPM). A reference that discusses these transport methods as well as other diffusion-based approaches is Cho [5].

The transmutation of nuclides during reactor operation is a time-dependent process that, in the context of lattice depletion, is coupled with the steady state neutronics solution of the scalar flux. A general expression for the change in the concentration $N$ of a nuclide $i$ by neuron interaction and radioactive decay is given as

$$
\frac{d N_{i}(t)}{d t}=\sum_{j=1}^{M} l_{i j} \lambda_{j} N_{j}+\bar{\phi} \sum_{k=1}^{M} f_{i k} \sigma_{k} N_{k}-\left(\lambda_{i}+\sigma_{i} \bar{\phi}\right) N_{i},
$$

where $l_{i j}$ is the fraction of radioactive disintegrations by nuclide $j$ leading to the formation of nuclide $i, \lambda$ is the radioactive disintegration for nuclide $i$, and $f_{i k}$ is the fraction of neutron reactions by nuclide $k$ leading to the formation of nuclide $i$. These coefficients including the microscopic reaction rate is assumed constant for a given computation cell, or cross section region (XSR), and for a given depletion period. The removal of the nonlinear dependence of the microscopic reaction rate, $\sigma \bar{\phi}$, on the nuclide concentration is often partially recovered through the use of predictor-corrector methods. 
There are five governing equations of fluid dynamics, one mass-conservation, one momentum-conservation in each of the three directions, and one energyconservation. The mass-conservation, also known as the continuity, equation is

$$
\frac{\partial \rho}{\partial t}+\nabla \cdot(\rho \vec{U})=0
$$

where $\vec{U}$ is the velocity field. In the case of constant density flows, the continuity equation reduces to the divergence-free condition of

$$
\nabla \cdot \vec{U}=0
$$

The incompressible Navier-Stokes equation represents momentum-conservation and is given as

$$
\rho \frac{D \vec{U}}{D t}=-\frac{1}{\rho} \nabla p+\nu \nabla^{2} \vec{U}
$$

where the material, or total or Lagrangian, derivative is the sum of the Eulerian, or fixed reference frame, derivative and the advective motion of a fluid particle,

$$
\frac{D \vec{U}}{D t}=\frac{\partial}{\partial t}+\vec{U} \cdot \nabla
$$

The kinematic viscosity in Equation 26 is defined as $\nu=\mu / \rho$. Together, the continuity and Navier-Stokes equations govern the flow of constant-property Newtonian fluids.

In addition to using CFD for the solution of the flow equations, 3-D heat conduction and associated heat removal through convection is solved, i.e. the simulataneous solution of heat transfer in both the solid (fuel) and fluid (coolant) is sought. This coupled thermal solution is known as conjugate heat transfer. The temperature equation in the fluid is given as

$$
\frac{\partial\left(\rho c_{p} T\right)}{\partial t}+\rho c_{p} \vec{U} \cdot \nabla T=\beta \vec{U} \cdot \nabla P+\nabla \cdot(K \nabla T)+q^{\prime \prime \prime} ;
$$

and the temperature equation in the solid is given as

$$
\rho c_{p} \frac{\partial T}{\partial t}=\nabla \cdot\left(k_{t h} \nabla T\right)+q^{\prime \prime \prime} .
$$

The standard wall function for convective heat transfer at the wall is

$$
q_{w}^{\prime \prime}=\frac{\rho_{f}(y) c_{p, f}(y) u^{*}\left(T_{f}-T_{w}\right)}{T^{+}\left(y^{+}(y)\right)},
$$

and the heat transfer coefficient is

$$
h(y)=\frac{\rho_{f}(y) c_{p, f}(y) u^{*}}{T^{+}\left(y^{+}(y)\right)},
$$


with $\rho_{f}$ being the density of the fluid, $c_{p, f}$ the specific heat of the fluid, $u^{*}$ the reference velocity, and $T^{+}$the non-dimensional temperature [4].

Both the lattice depletion and fluid dynamics physics are coupled to the physics of heat transfer through the fuel rod, given an internal volumetric heat source.

\subsection{Coupled Equations}

Because the high-fidelity prediction of CRUD deposition involves several complex and coupled solution methods, the most useful form to express the coupled equations is through the use of a specific physics operator. Such an operator simply takes inputs and transforms them to outputs. Of course, the inputs and outputs are not exchaustive, but are only included if relevant to the coupling of the physics. Moreover, a simplistic conformal mesh (one-to-one) solution transfer between the physics is assumed for this discussion; however, in the actual coupeld simulations, non-conformal solution transfers are performed.

The nuclide concentration $N_{j}$ is a vector that includes all relevant isotopes $j$, including, if applicable, specific CRUD and boron concentrations provided from the CRUD physics. All remaining quantities discussed in this section are scalars values for a single cell within the respective physics' spatial discretization at an instant in time. Also, the neutron flux and cross sections represent collapsed, single energy group values.

For the steady state neutronics physics, the inputs consist of the nuclide concentration, the nuclide microscopic cross section $\sigma$, and the nuclide temperature $T$ and the nuclide (coolant) density $\rho_{\text {cool }}$. The neutronics operator $\mathcal{N}$ respresents the solution of the 3-D transport equation, shown in Equation 19, resulting in the local flux distribution $\phi$ and the critical coolant boron concentration $B_{c o o l}$,

$$
\left(\phi, B_{\text {cool }}\right)=\mathcal{N}\left(\sigma, N_{j}, T, \rho_{\text {cool }}\right),
$$

where $T$ includes the coolant, cladding, and fuel temperatures. The power density distribution $Q^{\prime \prime \prime}$ is extracted and scaled to the flux distribution,

$$
Q^{\prime \prime \prime}=\mathcal{P}(\phi),
$$

and the neutron reaction rate with boron (isotope $b$ ) within the CRUD layer is

$$
X_{b}=\phi \sigma_{b} N_{b} .
$$

The nuclide depletion, often in the form of Equation 23, is more simply represented in terms of the coupled components as

$$
N_{j}=\mathcal{D}\left(\phi, \sigma_{j}\right) .
$$

The steady state neutronics and nuclide depletion physics are coupled through $N_{j}, \phi$, and $\sigma_{j}$.

The CFD physics is represented by two primary equations, the Navier-Stokes equations as shown in Equation 26 and the temperature equations as shown in Equations 28 and 29. The fluid-dynamic operator $\mathcal{F}$ takes the cladding heat 
flux $q^{\prime \prime}$ as input and gives the coolant temperature $T_{\text {cool }}$, coolant density $\rho_{\text {cool }}$, and turbulent kinetic energy $k$ distributions,

$$
\left(T_{\text {cool }}, \rho_{\text {cool }}, k\right)=\mathcal{F}\left(q^{\prime \prime}\right) .
$$

The heat transfer operator $\mathcal{H}_{s}$ gives takes the heat generation rate $Q^{\prime \prime \prime}$, coolant temperature $T_{\text {cool }}$, and CRUD thermal resistance $R_{c}$ as input to produce the solid temperature and heat flux conditions,

$$
\left(T_{\text {fuel }}, T_{\text {clad }}, q^{\prime \prime}\right)=\mathcal{H}_{s}\left(Q^{\prime \prime \prime}, T_{\text {cool }}, R_{c}\right) .
$$

The lattice depletion and CFD physics are coupled through $Q^{\prime \prime \prime}, T_{\text {fuel }}, T_{\text {clad }}$, $T_{\text {cool }}$, and $\rho_{\text {cool }}$.

The CRUD chemistry phsyics is represented by the operator $\mathcal{C}$, where inputs inlude the cladding temperature and heat flux, the coolant temperature and TKE, the coolant boron concentration, and the neutron reaction rate with boron. The outputs include the CRUD concentration $N_{c}$ and thermal resistance,

$$
\left(N_{c}, R_{c}\right)=\mathcal{C}\left(T_{\text {clad }}, q^{\prime \prime}, T_{\text {cool }}, k, B_{\text {cool }}, X_{b}\right) .
$$

The CFD and CRUD chemistry physics are coupled through $R_{c}, T_{\text {clad }}, T_{\text {cool }}, q^{\prime \prime}$, and $k$; whereas, the lattice depletion and CRUD chemistry physics are coupled through $B_{\text {cool }}, X_{b}$, and $N_{c}$.

The coupled variables between each of the physics is illustrated in Figure 6. The primary physics of lattice depletion, CFD, and CRUD chemistry are shown; additionally, the coupled sub-physics of (i) steady state neutronics and nuclide depletion, (ii) CFD fluid flow and solid heat transfer, and (iii) CRUD heat transfer and chemistry are also illustrated.

\subsection{Feedback Mechanisms}

The feedback mechanisms discussed in this section are the motivation for a coupled approach to CRUD deposition simulation. First, the primary feedbacks associated with the CILC and CIPS conditions are introduced. Then, the remaining secondary feedbacks are discussed. Because the amount of CRUD deposition does not correlate exactly with the amount of boron hideout within the CRUD, boron hideout may be expressed separately from the overall process of CRUD deposition.

Increased cladding temperature and heat flux leads to increased SNB, which increases the CRUD deposition rate. As CRUD builds up, there is an increased thermal resistance between the cladding surface and coolant, thus insulating the cladding and increasing the temperature. Higher temperature results in a more corrosive environment, possibly leading to CILC. Clearly, this is a positive feedback mechanism directly provided by the thermal hydraulics, as shown in Figure 7 (left).

On the other hand, as CRUD builds up and boron hideout occurs, the neutron absorbing properties decrease the power density. In turn, the heat flux is decreased, thus decreasing the CRUD and boron deposition rate. This is a 


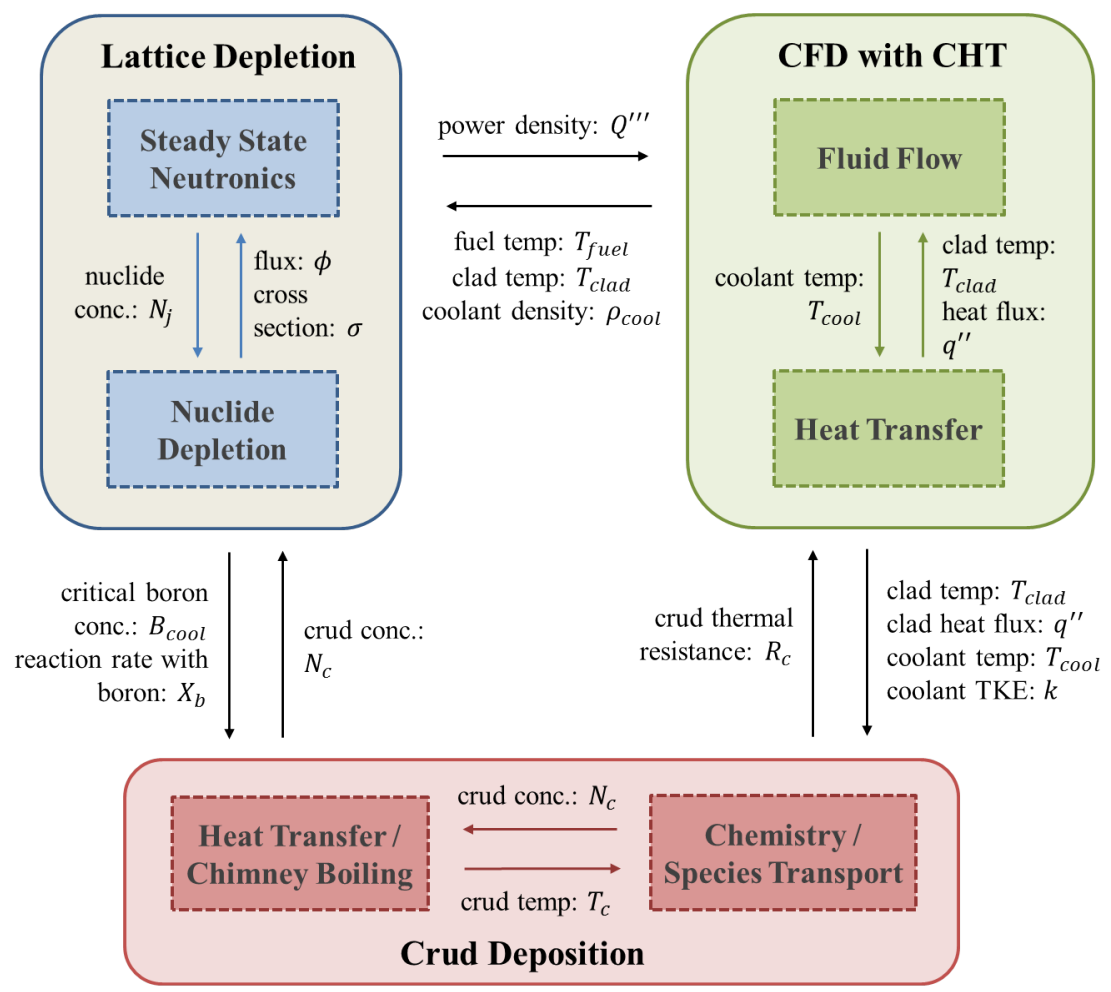

Figure 6: Multiphysics coupling and data exchange for high-fidelity CRUD deposition simulation.

negative feedback mechanism provided by the neutronics, but fed through the CFD to CRUD chemistry, as shown in Figure 7 (right). Localized suppressions of the power distribution caused by CRUD and boron hideout are defined as the CIPS condition.

The critical boron concentration within the coolant, as predicted by the neutronics, strongly influences the magnitude of boron hideout. The dissolved boric acid acts as a source for the deposition of boron within the CRUD layer. Therefore, another relevant feedback mechanism is that of increasing boron hideout, leading to increased localized neutron absorption, which decreases the overall multiplication factor $k_{e f f}$, or $k_{i n f}$ for systems modeled without radial leakage, of the reactor system. To compensate for this reduction, the critical boron concentration is also reduced, thus providing a negative feedback to the physics of boron hideout, as illustrated in Figure 8 (left).

An additional feedback associated with boron hideout is connected to the boron destruction rate caused by the neutron flux. The boron isotopes may undergo an $(n, \alpha)$ reaction, decreasing the boron concentration and increasing the lithium concentration according to 

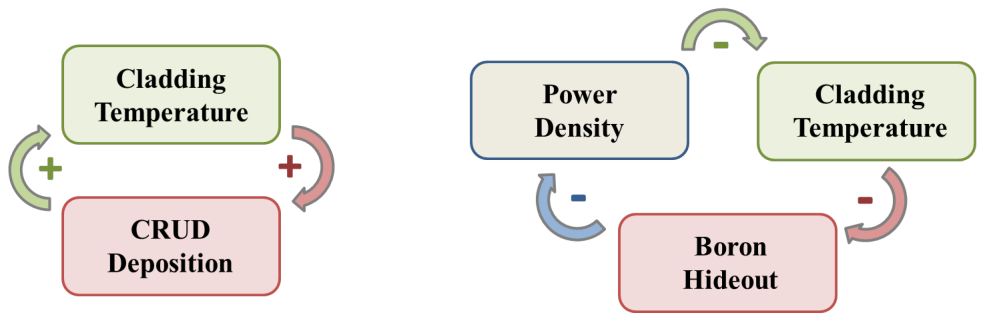

Figure 7: Positive feedback between CRUD deposition and CFD (left), and negative feedback with neutronics (right).
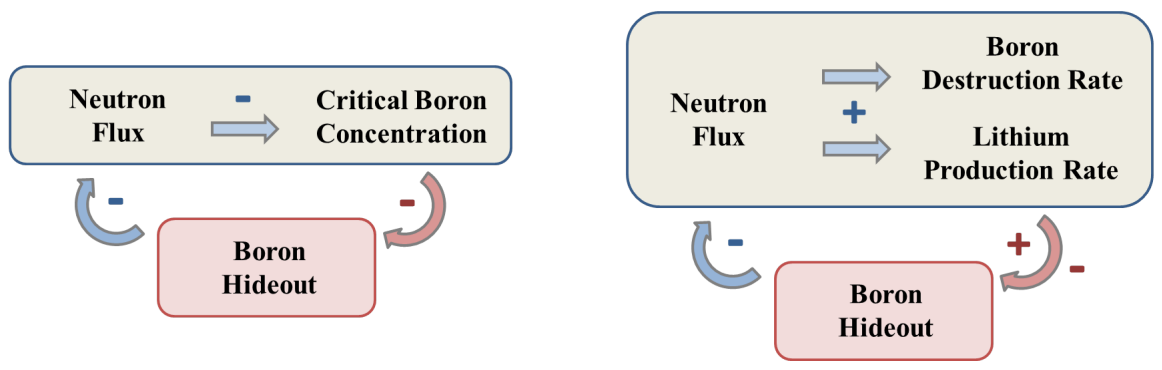

Figure 8: Feedbacks between CRUD chemistry and neutronics.

$$
{ }^{10} \mathrm{~B}+{ }^{1} n \rightarrow{ }^{7} \mathrm{Li}+\alpha .
$$

Both the boron and lithium concentrations within the coolant impact the degree of boron hideout, i.e. the production of lithium tetraborate, as described by the reaction in Equation 1. Depending on the chemistry conditions, including species concentration and CRUD temperature, this feedback may be positive or negative, as shown in Figure 8 (right). It should be noted that the production of lithium due to boron depletion is not considered in the simulations presented in this work.

Another vital component to realistic CRUD prediction is inclusion of the turbulence and flow swirl effects caused by the spacer grid mixing vanes. A high-fidelity thermal hydraulics tool, such as CFD, is necessary to capture the complicated turbulent flow patterns. The shear stress along the cladding surface, or alternatively the turbulent kinetic energy near the cladding wall, erodes the CRUD layer. The erosion is not necessarily a feedback because the CRUD condition does not significantly affect the turbulence effects; therefore, it is viewed as a boundary condition that is strongly dependent on the spacer grid mixing vane geometry. The magnitude of the erosion also decays exponentially downstream of the grid.

The distinct flow swirl around the fuel rod causes striping of the cladding temperature and TKE. However, these properties are anti-correlated, where a higher temperature exists where the TKE is lower, as depicted in Figure 9. This 


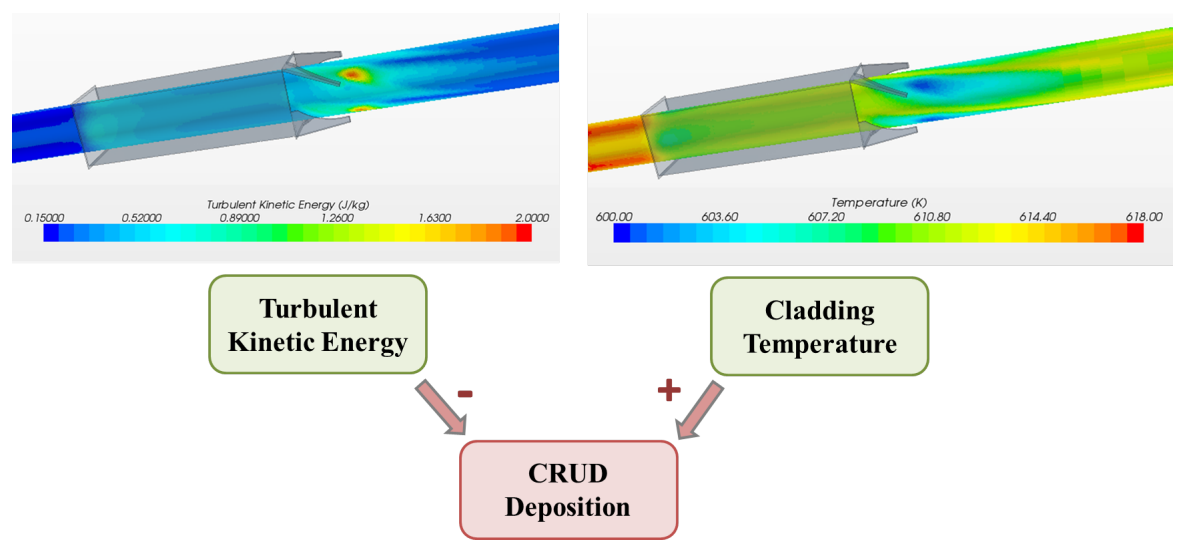

Figure 9: Anti-correlated TKE and cladding surface temperature caused by flow swirl induced by mixing vanes, including negative and positive CRUD deposition effects, respectively.

is attributed to degraded convective heat removal; naturally, these locations of higher cladding temperature, often referred to as hot spots, are the likely regions for SNB and subsequent CRUD deposition.

The accumulation of a CRUD layer on the cladding surface also influences the wall roughness, which is not accounted for in the current investigation.

\subsection{Temporal Coupling}

Figure 10 illustrates the temporal updates between the three codes. A uniform fixed-step scheme is employed to exchange data between the coupled physics, where the MAMBA solution lags the lattice depletion solution.

The DeCART/STAR-CCM + time stepping scheme consists of a fixed-point iteration (FPI). At each iteration, DeCART updates the power distribution on the basis of the fuel temperature and coolant density distributions computed by STAR-CCM+. Next, the STAR-CCM+ solution is updated based on the power density distribution supplied by DeCART. The iterations continue until the convergence criteria are satisfied. Currently, the coupled convergence criteria are a combination of the STAR-CCM + convergence criteria for continuity, z-momentum, and enthalpy, and the DeCART convergence criteria for the multiplication factor and the flux/power distribution.

After the coupled DeCART/STAR-CCM + solution has reached convergence, the cladding temperature, heat flux and TKE near the cladding wall is passed to MAMBA. Then, MAMBA advances its CRUD deposition solution in four hour time steps until reaching the end of the coarser time step length, $t_{\text {step }}$. In the following DeCART depletion calculation, the CRUD constituent concentrations at $t_{\text {half_step }}$ and $t_{\text {step }}$ are used. The coupled process repeats until the end of the operating cycle. 


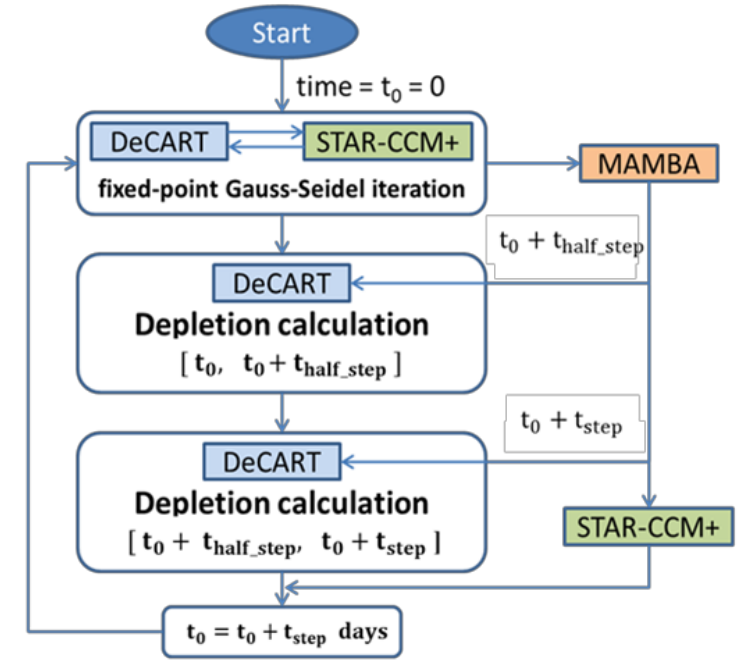

Figure 10: Temporal coupling between DeCART, STAR-CCM+, and MAMBA.

\section{Computational Models}

Three paritioned physics of lattice depletion, thermal hydraulics in the form of computational fluid dynamics, and CRUD and coolant chemistry are simulated using the computational tools of DeCART (Deterministic Core Analysis based on Ray Tracing), STAR-CCM+, and MAMBA (MPO Advanced Model for Boron Analysis). In Subsection 2.2, the modeling of CRUD chemistry and deposition kinetics within the code MAMBA was discussed in detail. The specific methods and models used within the remaining tools of DeCART and STAR-CCM + are discussed in this subsection.

\subsection{DeCART}

DeCART is a whole core neutron transport code capable of direct sub-pin level flux calculation at power generating conditions of a light water reactor. It requires neither a priori homogenization nor group condensation as needed in conventional reactor physics calculations. DeCART solves the 3-D neutron transport problem employing a 2D-1D method in which the planar 2-D solution is performed using the MOC solutions, and the axial 1-D solution is performed using the Nodal Expansion Method (NEM) based kernel. DeCART is capable of nuclide depletion via a two-block approach where the short- and long-lived nuclides are separately solved via the Bateman and matrix exponential methods, respectively. Additionally, the predictor-corrector and substep methods are available to enable longer depletion stepsizes. DeCART has been well validated for a wide range of pressurized and boiling water reactor applications (Hursin et al, 2008 and Joo et al, 2004).

The DeCART model domain is divided into a user-specified number of flat source regions (FSR), which are sufficiently small so that the sources, as well 

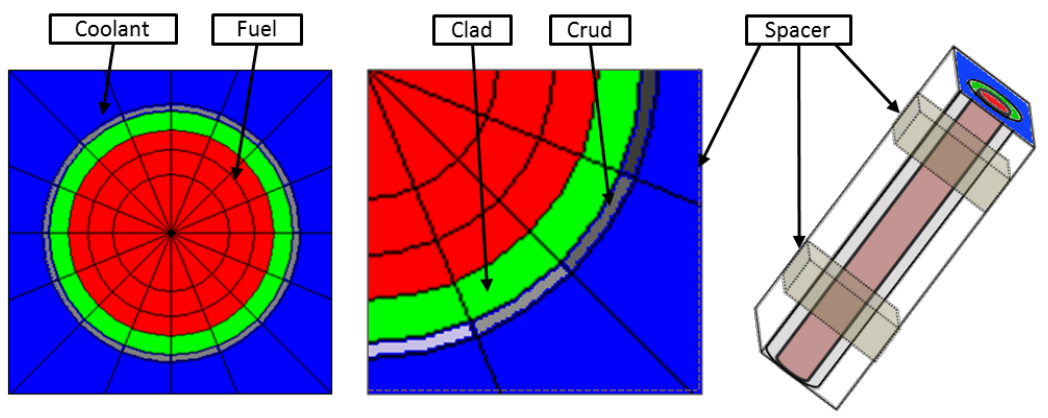

Figure 11: 2-D DeCART mesh showing material regions (left and center), and spacer grid model as boundary around pin cell (right).

as the material properties, are regarded constant in each region. Within each FSR, the angular flux and the corresponding scalar flux are determined and used to resolve the spatial flux distribution. To model CRUD, DeCART was modified to accept 3-D CRUD compositions from MAMBA and deposit these nuclides in a user-specified radial region with constant thickness of $\sim 150 \mu \mathrm{m}$, see Figure 11. To maintain a fixed spatial discretization in DeCART, the CRUD is homogenized with the coolant within this region reserved for CRUD. The CRUD concentration is allowed to vary circumferentially around the rod.

The DeCART model consists of a 3-D pin cell with an active fuel height of $365.76 \mathrm{~cm}$ and $20 \mathrm{~cm}$ thick water reflectors at each end. The radial boundary conditions are reflective, while the axial boundary conditions are vacuum. The power of the fuel pin is $107.17 \mathrm{~kW}$, which corresponds to a peaking factor of about 1.6 for a typical $17 \times 17$ PWR fuel assembly pin. Eight $4 \mathrm{~cm}$ tall grid spacers with $0.24 \mathrm{~mm}$ thick walls are included to simulate the neutron absorption effects. The fuel is composed of $\mathrm{UO}_{2}$ with $3.3 \mathrm{wt} \%$ enriched uranium-235. The cladding and grid spacer material is Zircaloy. A fuel-cladding gap is not included in the model. The coolant boron concentration (CBC) is letdown during depletion from $1950 \mathrm{ppm}$ to $5 \mathrm{ppm}$. Because the CBC is an important factor in boron hideout, the simulation attempts to maintain a semi-realistic boron letdown curve under high-power conditions, while allowing the multiplication factor to evolve as necessary with depletion. Figure 11 shows the pin cell geometry with select spacer grids and all flat source regions: 48 within the fuel and 16 within each of the cladding, CRUD, coolant, and grid spacer regions.

\subsection{STAR-CCM+}

STAR-CCM + is a commercial computational fluid dynamics code that solves the balance equations for continuity, 3-D momentum, and fluid enthalpy on a finite-volume 3-D mesh. STAR-CCM+ is capable of modeling conjugate heat transfer as described in Subsection 3.1, such that the conduction process is modeled within the fuel pellet and cladding and the convective heat transfer is modeled in the cooling fluid that surrounds the fuel pin. In the simulations 
presented here, the Reynolds-Averaged Navier-Stokes (RANS) approach for the solution of the standard $k-\varepsilon$ transport equations is used for the turbulence modeling of a single phase fluid [1]. Whereas, localized internal boiling within the CRUD layer is considered in the MAMBA code by Equation 3. The spatial-scale (microns) at which the CRUD layer develops is much smaller than typical CFD meshes. Therefore, in STAR-CCM+, the CRUD layer is modeled as a thermal resistance between the cladding and coolant.

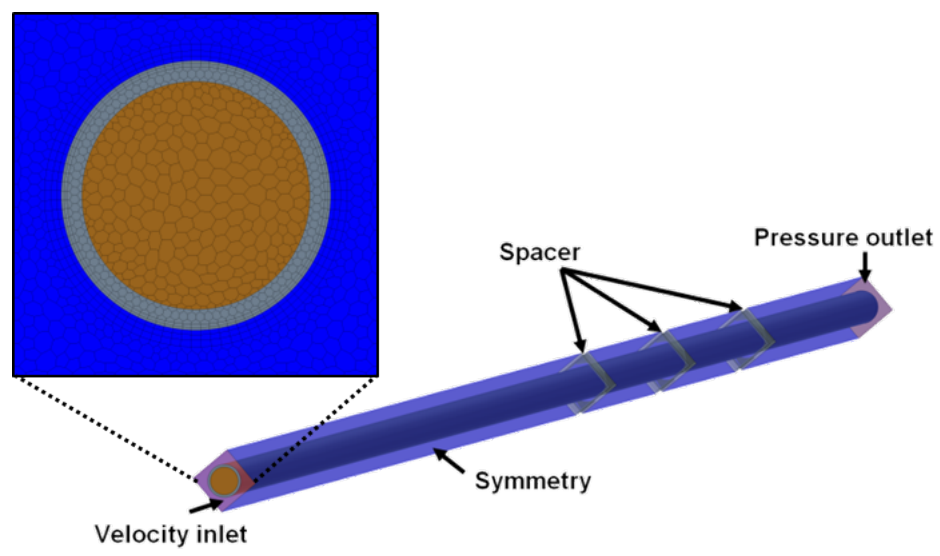

Figure 12: STAR-CCM+ geometry and mesh model showing boundary conditions and spacer grid locations.

The CFD domain includes the solid structure containing the fuel pellets and cladding, the water domain in the subchannel surrounding the fuel pin, and three spacer grids with mixing vanes. Only three of the eight grid spacers are included in the CFD model to reduce computation time. An inlet velocity of $5.278 \mathrm{~m} / \mathrm{s}$, inlet temperature of $556.76 \mathrm{~K}$, system pressure of $15.51 \mathrm{MPa}$, and pressure boundary condition is imposed for the inlet and outlet axial planes of the coolant domain, respectively. Symmetric boundary conditions are imposed on the lateral surfaces of the water domain. No-slip conditions are imposed on the spacer grids, pin walls, and outer cladding surface. A volumetric power source is used in the fuel domain (provided by the neutronics solution). The coolant density is calculated according to the following third-order polynomial:

$$
\rho_{\text {cool }}(T)=-1.16905 \times 10^{-5} T^{3}+1.225 \times 10^{-2} T^{2}-4.84697 T+1.670326 \times 10^{3} .
$$

The $\mathrm{UO}_{2}$ fuel and cladding thermal conductivity are set constant at 3.0 and 17 $\mathrm{W} / \mathrm{m}-\mathrm{K}$, respectively. The fluid-dynamic simulation includes conjugate heat transfer for the calculation of the temperature distribution in the fuel and cladding domains.

The CFD domain was meshed by means of polyhedral cells. The spacer grid regions, which rest at $203.28 \mathrm{~cm}, 249.00 \mathrm{~cm}$, and $294.72 \mathrm{~cm}$ from the bottom of the active fuel, were discretized using polyhedral cells with three layers of prismatic cells at the wall, as shown in Figure 2. The computational mesh upstream 
Table 1: STAR-CCM+ model mesh properties.

\begin{tabular}{|c|c|c|c|}
\hline Region & Cells & Faces & Vertices \\
\hline \hline Fuel & 430,803 & $2,642,496$ & $2,214,928$ \\
\hline Cladding & 454,521 & $1,915,847$ & $1,317,250$ \\
\hline Fluid & 776,970 & $3,401,427$ & $2,283,715$ \\
\hline Total & $1,662,294$ & $7,959,770$ & $5,869,893$ \\
\hline
\end{tabular}

and downstream of the grid spacers was generated by extrusion, applying the hyperbolic tangent law. Approximately 1.6 million cells makeup this model; additional mesh properties are summarized in Table 1 . The convergence criteria were fixed at $1 x 10^{-6}$ for continuity, z-momentum, and energy, respectively. Figure 12 shows the boundary conditions and the cross section mesh.

\section{3. $M A M B A$}

The 3-D MAMBA model domain consists of the outer cladding surface and a time-evolving radial CRUD region that grows toward the coolant. The radial mesh of the CRUD region adapts to the CRUD growth in $5 \mu \mathrm{m}$ increments. The azimuthal mesh consists of 16 uniform regions, aligning with the DeCART mesh. The pin is axially discretized into 74 uniform regions of $5 \mathrm{~cm}$.

The CRUD parameters include a user-defined initial porosity of 0.7 (which evolves in time), solid density of $5.33 \mathrm{~g} / \mathrm{cm}^{3}$, solid thermal conductivity of 0.011 $\mathrm{W} / \mathrm{cm}-\mathrm{K}$, a chimney radius of $4 \mathrm{\mu m}$, chimney density of $1.6 x 10^{5} \# / \mathrm{cm}^{2}$, and a chimney heat transfer coefficient of $2.12 x 10^{6} \mathrm{~W} / \mathrm{m}^{2}-\mathrm{K}$. The nickel ferrite surface deposition rate is given by an Arrhenius rate expression, as shown in Equation 8, where and $A=110.0 \mathrm{~cm} / \mathrm{s}$ and $E=10 \mathrm{kcal} / \mathrm{mole}$. The CRUD growth rate multiplier, due to $\mathrm{SNB}$ is set to $1.50 x 10^{-3} \mathrm{~cm}^{3} / \mathrm{J}$. The coolant species concentrations are also fixed for soluble nickel, soluble iron, and particulate as $0.22 \mathrm{ppb}, 1.32 \mathrm{ppb}$, and $1.76 \mathrm{ppb}$, respectively. The same coolant boron concentration letdown as described for the DeCART model is imposed. The coolant lithium concentration is correlated with the coolant boron concentration and is defined in Figure 13.

\section{Coupled Results}

\subsection{Beginning of Cycle Solution}

Prior to depletion and CRUD deposition, the beginning of cycle (BOC) converged solution is found by a coupled DeCART/STAR-CCM + simulation. The converged core characteristics are an effective multiplication factor of 1.13099 and an axial offset of $+4.15 \%$; this is the starting point for the 500-day depletion simulation. The radially-integrated, converged axial power distribution (left) and average CRUD region (without CRUD at BOC) temperature distribution (right) are shown in Figure 14. The locations of the eight spacers grids in DeCART, and the associated power depressions due to the increased neutron 


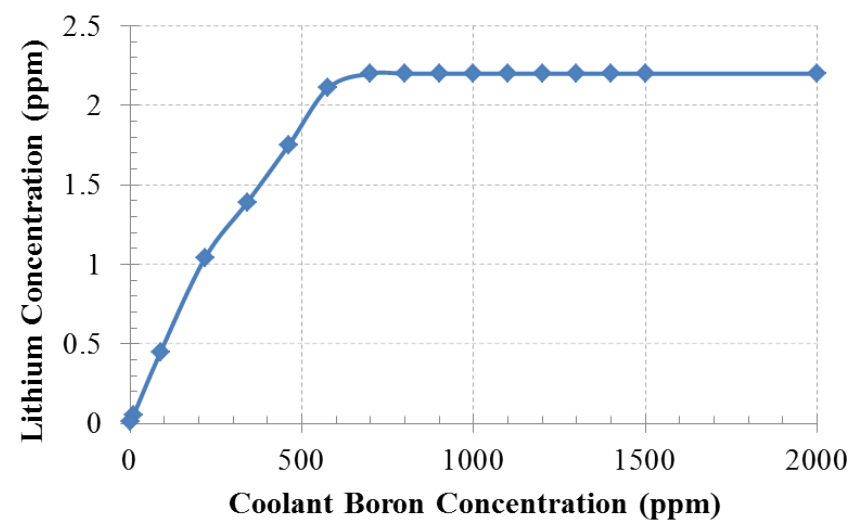

Figure 13: Coolant lithium concentration correlation with coolant boron concentration within MAMBA.

absorption by the grid material, are labeled with arrows. The purple, bolded arrows represent the locations of the three grid spacers and mixing vanes, which are modeled with STAR-CCM+.

The average temperature within the DeCART cells (reserved for CRUD deposition), which are initially filled with coolant, increases approximately 50 kelvin along the axis of the fuel pin, as shown in Figure 14 (right). The substantial increase in heat removal, due to the increase in turbulent mixing following the three spacer grids with mixing vanes, is demonstrated by the depressions in the temperature. The power density (left) and temperature (right) of each DeCART cell at the core mid-plane (core elevation of $206.59 \mathrm{~cm}$ ) for the converged DeCART/STAR-CCM + solution is shown in Figure 15. The power density increases radially from the fuel center due to spatial self-shielding. The increased power and burnup within the outer regions of a fuel pin is often referred to as rim effect. With depletion, plutonium build-up occurs in this region; however, a finer radial mesh within the fuel is required to fully capture this effect. Figure 15 also shows a small azimuthal variation in the fuel and cladding temperature caused by the turbulent mixing of the coolant modeled by STAR-CCM+; however, the neutronics effects of these temperature variations is negligible.

\subsection{Global CRUD Characteristics}

The integral CRUD mass, maximum CRUD thickness and integral boron mass deposited along the fuel rod during the cycle depletion are shown in Figure 16. As the CRUD thickens, internal boiling also increases, which concentrates the soluble boron and lithium species near the cladding surface. The combination of high temperature and species concentration leads to precipitation of lithium tetraborate. As a result, the boron within the CRUD layer quickly increases to $9.87 \mathrm{mg}$ between 250 and $400 \mathrm{EFPD}$. After $400 \mathrm{EFPD}$, the total boron mass decreases to $8.84 \mathrm{mg}$ due to diffusion of species out of the CRUD 


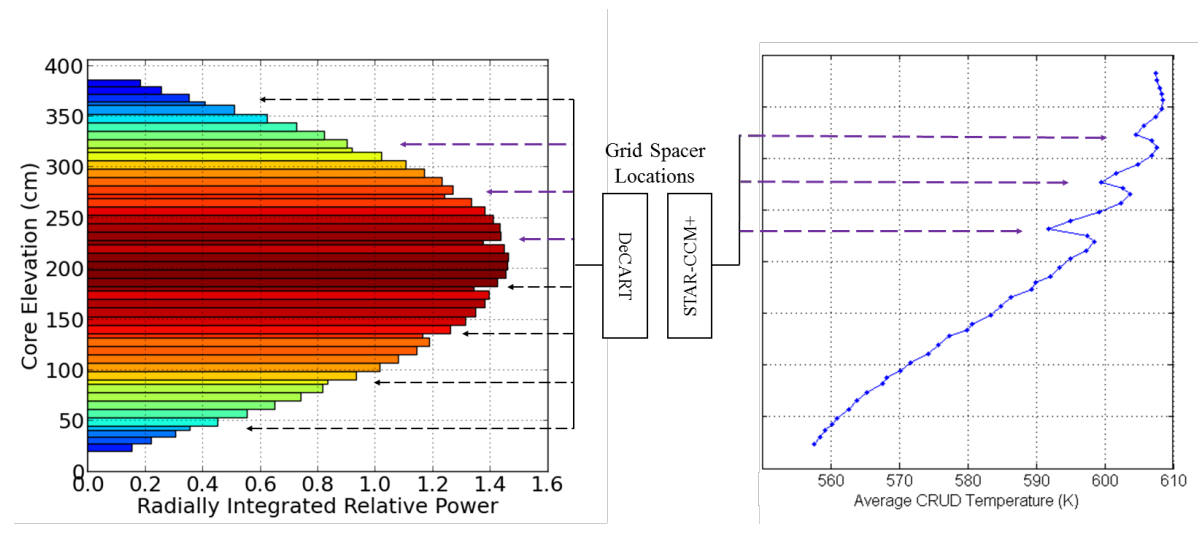

Figure 14: BOC power (left) and designated CRUD region temperature distribution (right), including spacer grid effects.

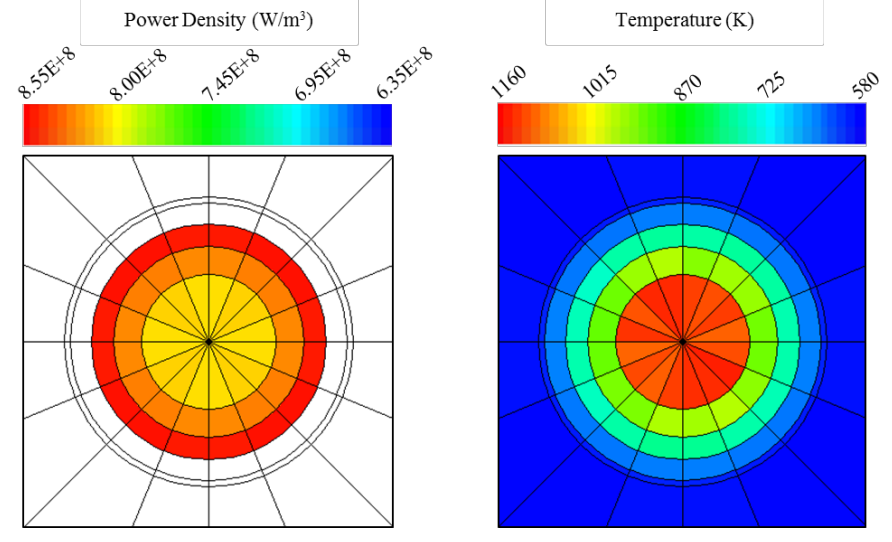

Figure 15: Planar power density and temperature distributions at BOC within DeCART mesh. 

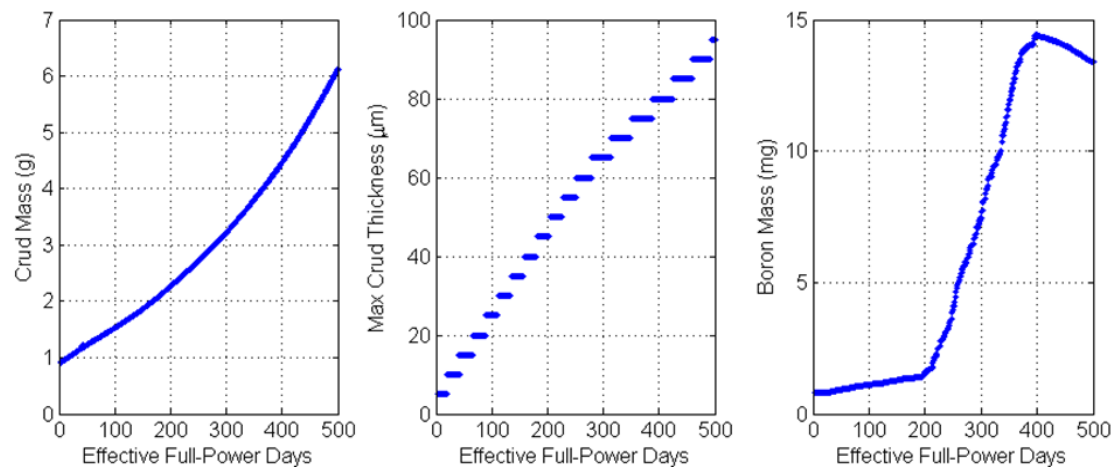

Figure 16: Global CRUD mass (left), thickness (center), and boron mass (right).

layer, boron destruction caused by neutron flux, and reduced coolant boron concentration feeding the CRUD layer. At the end of cycle (EOC), the integral CRUD mass and local thickness reaches maximum values of $5.93 \mathrm{~g}$ and $90 \mu \mathrm{m}$, respectively.

\subsection{CRUD Striping Effect}

The flow swirl induced by the mixing vanes is the primary contributor to the strong azimuthal variation of the CRUD deposition process. The turbulent kinetic energy and outer cladding wall temperature are anti-correlated, where an increase in TKE increases the convective heat transfer, leading to a decrease in the outer cladding surface temperature. The localized increase in TKE is shown in Figure 9 (left). Each of the four vanes produces a region of very high relative turbulence, which effectively decreases the cladding temperature. Figure 9 (right) shows that a temperature difference of more than 10 kelvin occurs within just a few millimeters. Consequently, the azimuthal variation of these properties contributes to a characteristic striping pattern of CRUD deposition.

Figures 17 and 18 show the predicted cladding-CRUD interface temperature and the CRUD-coolant interface temperature $10 \mathrm{~cm}$ after the second spacer grid and $10 \mathrm{~cm}$ before the third spacer grid, respectively. Further downstream of the second spacer grid, CRUD build-up increases due to higher coolant temperatures and less CRUD erosion. At $10 \mathrm{~cm}$ downstream of the second spacer, the CRUD layer shows a temperature difference of approximately four degrees at locations experiencing the lowest TKE. On the other hand, at $10 \mathrm{~cm}$ before the third spacer (within the same span), the CRUD layer shows a temperature drop of more than eight degrees in some locations.

The CRUD thickness and boron surface density distributions are shown in Figure 19. The effects of the grid spacers and mixing vanes are demonstrated. CRUD deposition is nearly non-existent immediately following each grid spacer, which is a consequence of the erosion correlated to the intensity of the TKE. As previously discussed, the TKE reduces the outer cladding temperature and 


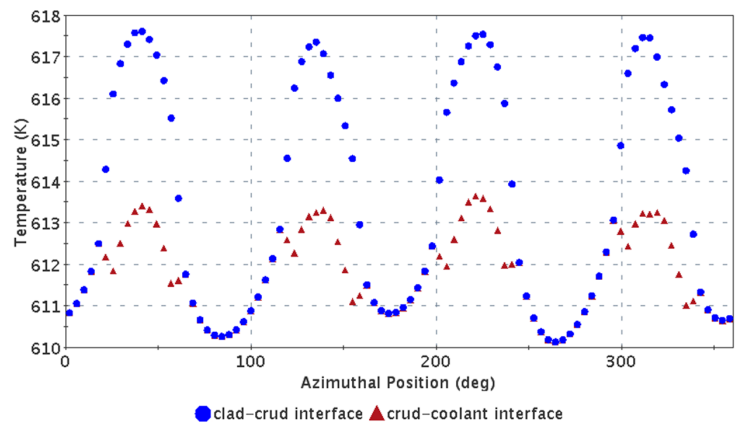

Figure 17: Cladding-CRUD and CRUD-coolant interface azimuthal temperature distribution $10 \mathrm{~cm}$ after second spacer grid at 300 EFPDs.

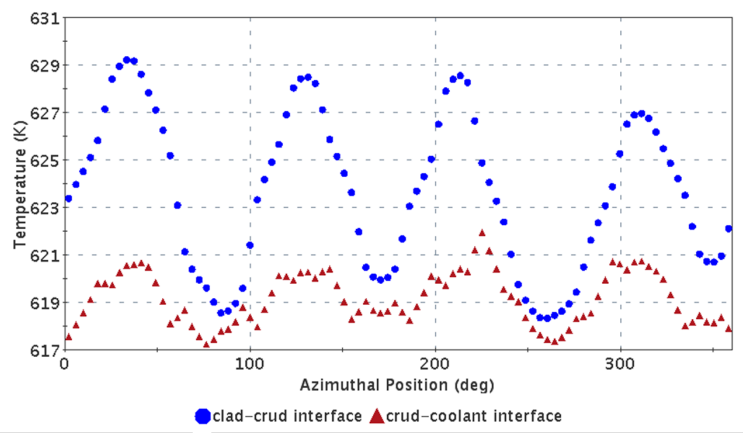

Figure 18: Cladding-CRUD and CRUD-coolant interface azimuthal temperature distribution $10 \mathrm{~cm}$ before third spacer grid at 300 EFPDs.

therefore decreases the probability of CRUD deposition and internal boiling. The TKE is also correlated with the erosion of the CRUD layer. Therefore, three regions exist where CRUD is nearly absent, one for each grid spacer modeled with STAR-CCM+.

The combination of the erosion and azimuthal temperature variation yields the striped deposition pattern. The effect of the grid spacers on the CRUD deposition pattern has more thoroughly been investigated [15]. Once lithium tetraborate precipitation begins to occur (after 250 EFPD in this simulation), as shown in Figure 19, middle and right, localized regions of very high boron concentration develop.

\subsection{Local CRUD Characteristics}

The results in this subsection are shown in two-dimensional plots of the CRUD layer distribution as a function of radius (from the outer cladding surface) and active core elevation. The azimuthal region is fixed and corresponds to the MAMBA azimuthal node spanning $0^{\circ}$ and $22.5^{\circ}$, referred to as node $11.25^{\circ}$. 


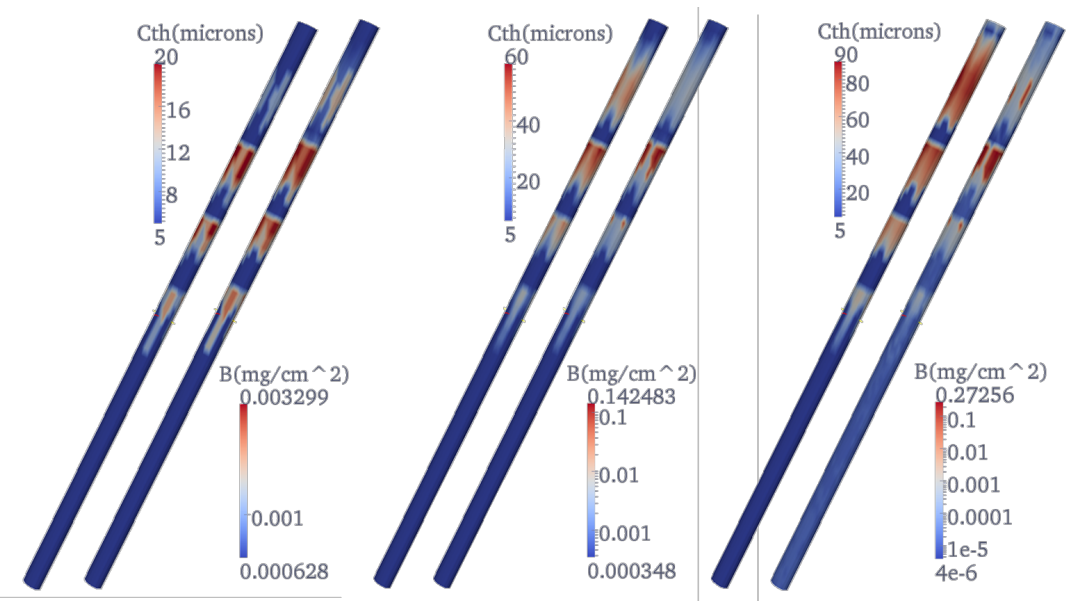

Figure 19: CRUD thickness and boron surface density along lenth of fuel rod at 100, 300, and 500 EFPDs.

Three burnup states are shown in each figure, $6.27,18.82$, and $31 \mathrm{MWd} / \mathrm{kgHM}$; these correspond to 100, 300, and 500 EFPD.

The boron density within the CRUD layer is shown in Figure 20. The precipitation occurs locally in one axial position; however, as shown in Figure 19, four of the regions are present circumferentially around the rod. The precipitation threshold, controlled by temperature and the concentration of the boric acid and lithium within the coolant, is exceeded at these locations. This is attributed to local SNB that pulls coolant into the CRUD and to the cladding surface, concentrating the boric acid and lithium species during vaporization. Once precipitation occurs, the dense boron creates a localized neutron sink, which jointly reduces the power and temperature of the cladding surface. The effect of the grid spacer mixing vanes on the CRUD build-up is also evident in Figure 20.

Figure 21 shows the evolution of the CRUD temperature. As the CRUD thickens along the upper regions of the fuel pin cladding, heat transfer from the solid rod to the coolant deteriorates, leading to local rises in the temperatures. Consequently, the possibility of SNB increases as does the likelihood of CILC and fuel failure. Figure 22 shows the porosity within the CRUD layer. In general, the outer regions of the CRUD layer (closest to the bulk fluid) are the most porous. The inner regions (closest to the cladding), where internal boiling occurs, are the densest. The densest regions are a result of the precipitation. Between an active core elevation of 280 and $290 \mathrm{~cm}$, the dense is visible for 300 and 500 EFPD.

\subsection{CRUD Induced Power Shift}

According to the Nuclear Regulatory Commission (NRC) guidelines, maintaining sufficient operating margins is a requirement of the operating utility. 

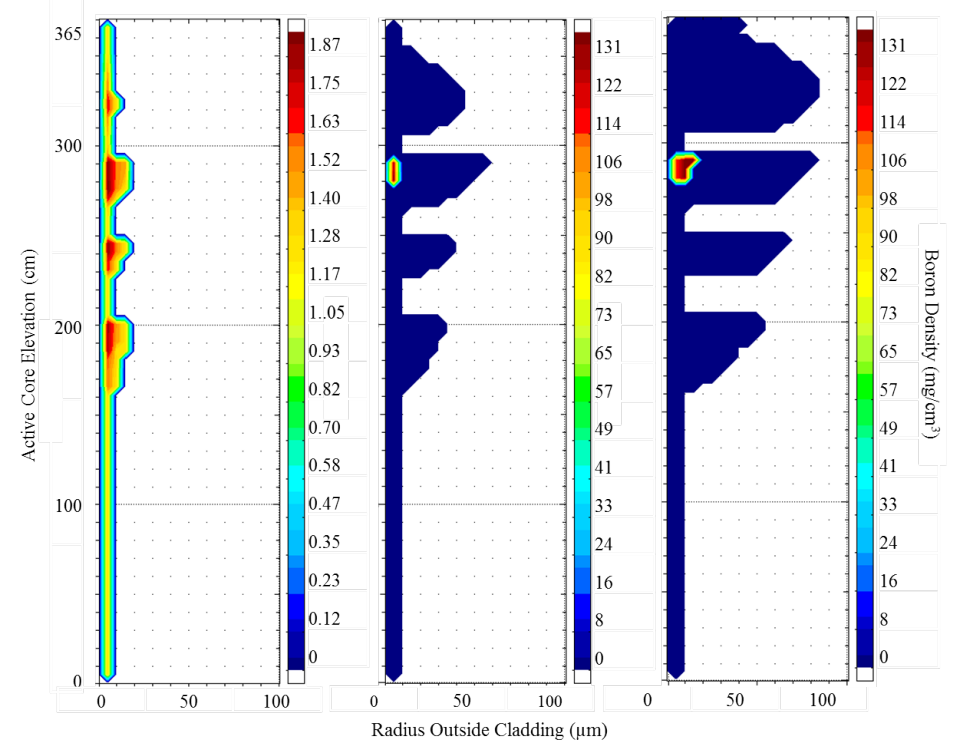

Figure 20: CRUD distribution along fuel rod shaded with the internal boron density distribution at 100, 300, and 500 EFPDs.

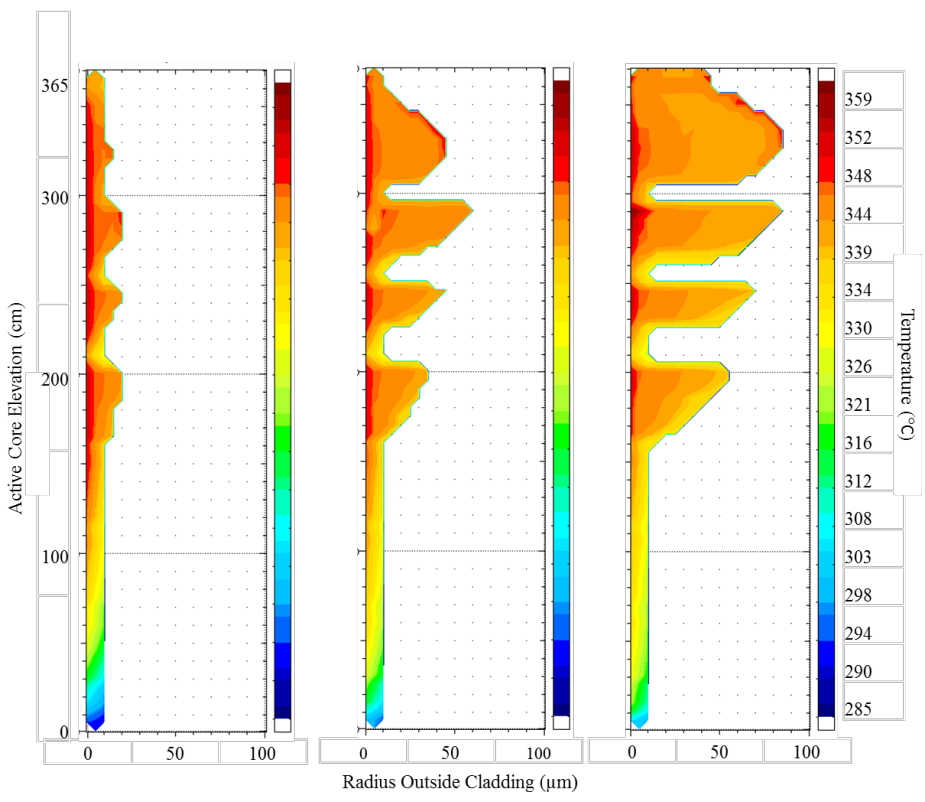

Figure 21: CRUD distribution along fuel rod shaded with the internal temperature distribution at 100,300 , and 500 EFPDs. 


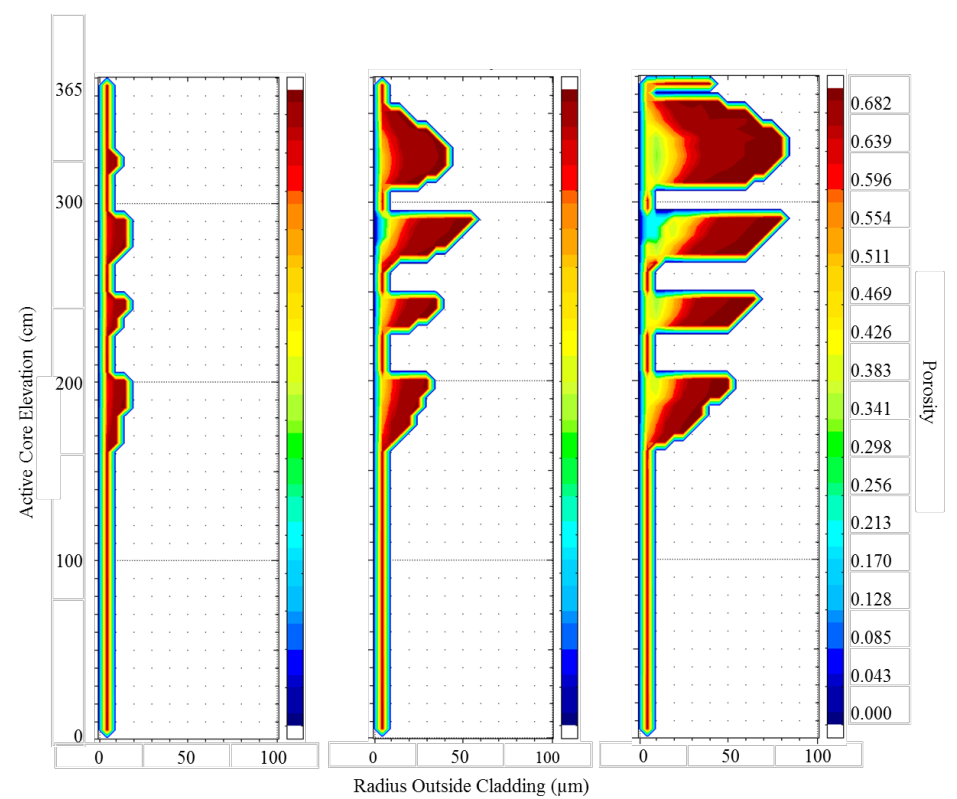

Figure 22: CRUD distribution along fuel rod shaded with the internal porosity distribution at 100,300 , and 500 EFPDs.

CRUD build-up alters the power distribution within the reactor core from the expected. In this simulation, the expected distribution at 300 EFPDs is shown in Figure 23 (top-left). The neutron absorption effects of the grid spacer materials are demonstrated for the eight spacers included in the DeCART model. The highest power regions of the fuel, near the core center, experience the greatest burn. Thus, with time, the power distribution flattens, and eventually develops a double-hump profile.

Clear differences in the expected power distribution and CIPS power distribution in Figure 23 (top-right), where localized reductions in power are seen in the upper regions, corresponding to the axial location of lithium tetraborate precipitation. The boron-10 in the dense precipitate pushes the power distribution toward the bottom of the core. Fuel burnup favors the bottom-peaked core power and contributes to flattening the distribution by the end of cycle (not shown). Figure 23 (bottom) displays the difference in power at each axial elevation between the expected and CIPS results. Generally, a power reduction occurs in regions of CRUD deposition, and a subsequent power increase occurs in the less affected regions. Because these plots show differences in relative pin powers, a decrease in power in one region must be balanced by an increase in another. The power shift caused by CRUD build-up and boron hideout causes a $10 \%$ difference in relative planar power near $310 \mathrm{~cm}$ core elevation.

The CIPS condition is a core-wide (global) phenomenon and the axial offset of the power is strongly dependent on core characteristics that evolve with bur- 

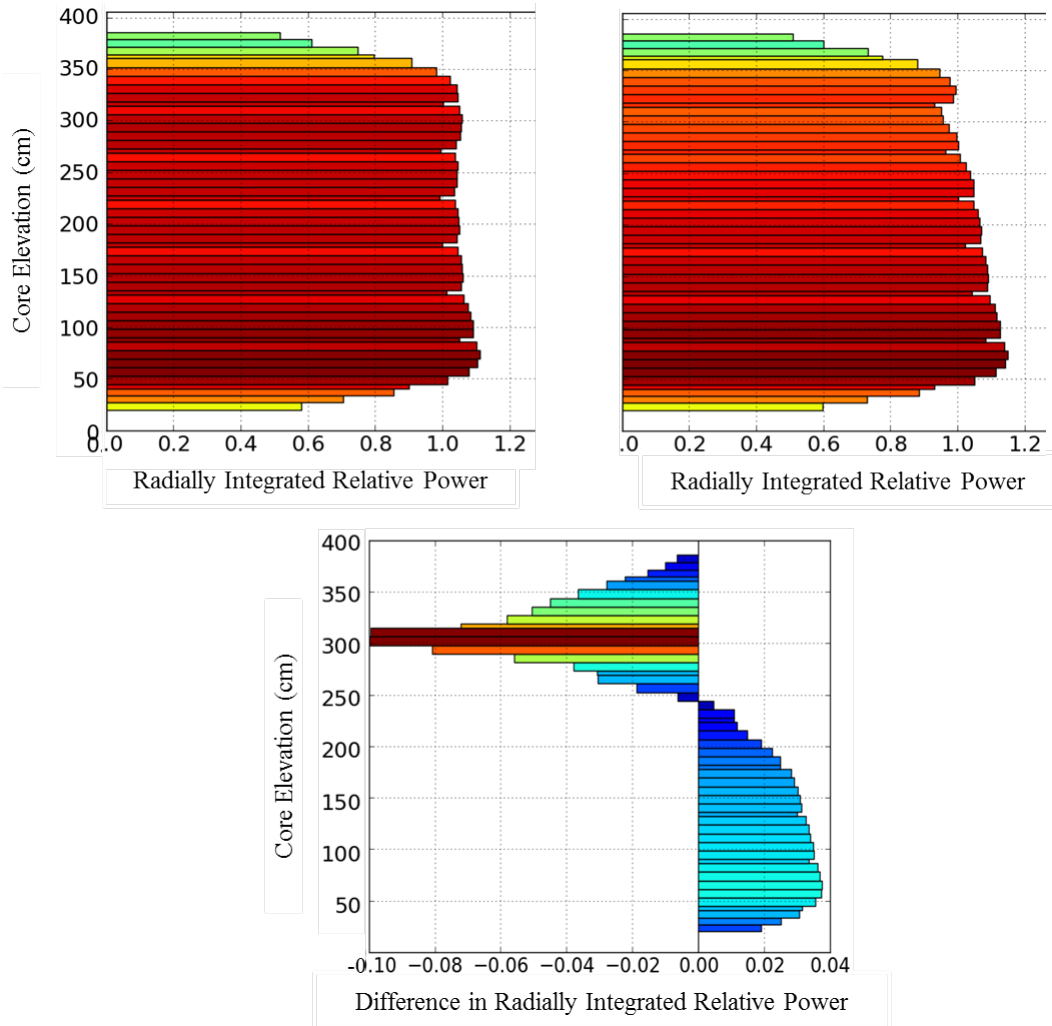

Figure 23: Axial power distribution comparisons at 300 EFPD for no-CRUD (top-left), with CRUD (top-right) showing CIPS, and difference in powers (bottom).

nup, such as moderator temperature coefficient, coolant boron concentration, and fuel enrichment. Therefore, realistic modeling of a single radially reflective fuel pin is challenging without the presence of burnable absorbers, burned fuel, etc. Nonetheless, the AO, which is calculated as the ratio of the difference between the integrated power in the top and bottom halves of the core and the sum of these integrated powers, is shown in Figure 24 for demonstration purposes. The small amount of CRUD deposition, completely in the form of nickel ferrite, in the first several $\mathrm{MWD} / \mathrm{kgHM}$ leads to a slightly more negative AO than the no CRUD case. However, this difference is slight and varies due to the coolant boron concentration. Once lithium tetraborate precipitation occurs (beginning around $15 \mathrm{MWD} / \mathrm{kgHM}$ ), a negative power shift begins. By a core burnup of $22.5 \mathrm{MWD} / \mathrm{kgHM}$, the AO magnitude reaches its maximum of $7.0 \%$. A combination of the reduction of the CBC, diffusion of species out of the CRUD layer, and boron destruction lead to a net reduction in the boron hideout and a less negative AO by EOC. Additionally, the bottom-favored power distribution increases the fuel burnup in these regions, which contributes toward pushing the power distribution closer to $0 \%$ axial offset. 


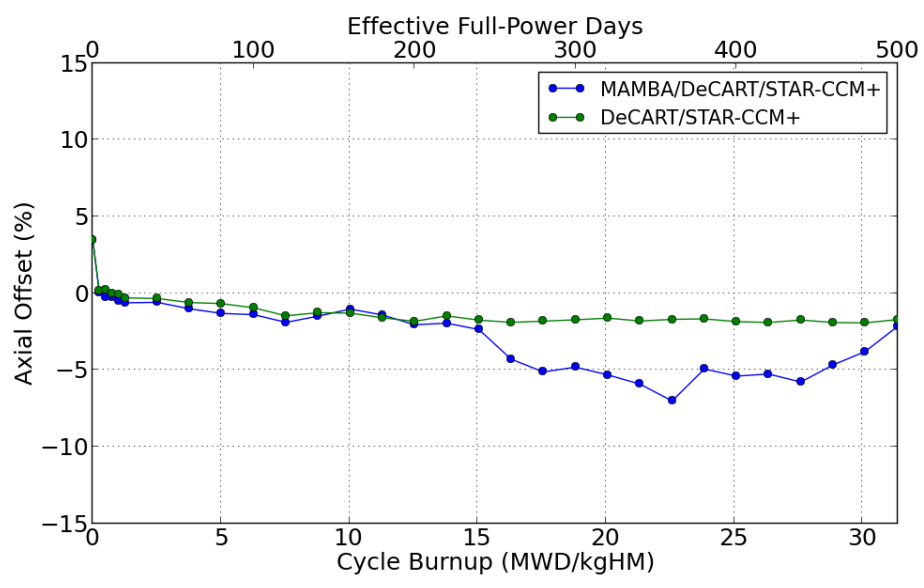

Figure 24: Axial offset of power distribution comparison.

\section{Computational Performance}

DeCART and STAR-CCM + were executed on an IBM x3755 M3 server. The server features $642600 \mathrm{MHz}$ cores (4xCPU AMD Opteron 6282SE 16 core) and 96 gigabytes of RAM. The DeCART simulations were run using 51 MPI processes, and STAR-CCM + was run in parallel with 18 processes. The MAMBA model was simulated on a Linux workstation, featuring 12 Intel Xeon $2670 \mathrm{MHz}$ cores and 12 gigabytes of RAM. A single processor is used for the single fuel rod. The total run and CPU time statistics are summarized in Table 2. The total simulation wall clock time was 29.8 hours, where $37 \%$ of the time was spent in MAMBA, $10 \%$ spent in DeCART depletion and 19\% spent in DeCART steady state, and 34\% in STAR-CCM + . However, the actual CPU time was 633 hours, where $2 \%$ of the time was spent in MAMBA, $24 \%$ spent in DeCART depletion, $45 \%$ spent in DeCART steady state, and $29 \%$ in STAR-CCM+. MAMBA carries the least computational expense and scales well because each fuel pin can be independently simulated on a single processor. Despite DeCART depletion and steady state using nearly $70 \%$ of the CPU time, the CFD simulation will prove to be the limiting factor as the model size increases.

\section{Conclusions}

A computational framework for the high-fidelity simulation of CRUD deposition has been developed as an investigative tool to better understand the physics and feedbacks associated with CRUD deposition. The framework includes the coupled physics of lattice depletion, computational fluid dynamics, and CRUD chemistry. The codes DeCART, STAR-CCM+, and MAMBA were coupled together via the operator-splitting technique, and were used to simulate a single fuel pin, depleted through a 500-day cycle. 
Table 2: Computational requirements of coupled simulation.

\begin{tabular}{|c|c|c|c|c|}
\hline $\begin{array}{c}\text { Code } \\
\text { Segment }\end{array}$ & $\begin{array}{c}\text { Total Wall } \\
\text { Time (hr) }\end{array}$ & $\begin{array}{c}\text { Fraction of } \\
\text { Total Wall } \\
\text { Time (\%) }\end{array}$ & $\begin{array}{c}\text { Total CPU } \\
\text { Time (hr) }\end{array}$ & $\begin{array}{c}\text { Fraction of } \\
\text { Total CPU } \\
\text { Time (\%) }\end{array}$ \\
\hline \hline MAMBA & 11.0 & 37.0 & 11.0 & 1.70 \\
\hline $\begin{array}{c}\text { DeCART } \\
\text { Depletion }\end{array}$ & 2.97 & 9.90 & 151 & 23.9 \\
\hline $\begin{array}{c}\text { DeCART } \\
\text { Steady }\end{array}$ & 5.65 & 19.0 & 288 & 45.0 \\
\hline $\begin{array}{c}\text { STAR- } \\
\text { CCM+ }\end{array}$ & 10.2 & 34.1 & 183 & 29.4 \\
\hline
\end{tabular}

Recreation of the classic striped CRUD pattern suggests the coupled physics qualitatively behave as expected. The inclusion of a high-fidelity fluid flow model, such as those provide by CFD, is a vital component to accurate CRUD deposition predictions. The spacer grid mixing vanes induce a flow swirl that results in cladding hot spots, where SNB, and thus CRUD build-up, is more likely. Moreover, the increased turbulence caused by the vanes is strongly correlated with the CRUD deposition pattern because the CRUD may be eroded.

Data reported by EPRI suggests that a core average boron build-up of 0.005 pounds $(2,267.96 \mathrm{mg})$ per assembly corresponds to a $5 \%$ AO difference (EPRI, 2004). The $9.87 \mathrm{mg}$ of boron predicted at $400 \mathrm{EFPD}$ in this simulation, extrapolated to a $17 \times 17$ assembly with 264 fuel rods (assuming equal CRUD loading), gives 0.00575 pounds $(2,605.68 \mathrm{mg})$. The difference in the axial offset predicted in Figure 24 is approximately $5.5 \%$, which is proportionally comparable to the expected AO (EPRI, 2004). This comparison supports the global rate of CRUD growth and boron hideout is within expectation. By no means does this comparion verify or validate the coupling framework, but it at least demonstrates a basis for additional investigations of the physics and modeling parameters.

The initial implementation of the framework may be improved by utilizing more advanced solution transfer methods and expanding the functionality of the MAMBA code. Solution transfer improvements include (i) eliminating the timelag between the nuclide depletion and CRUD deposition by employing a P-C or Crank-Nicolson scheme, (ii) interpolation of the exchanged data parameters to eliminate non-conformal mesh deficiencies, and (iii) adding more physics-based convergence criteria to ensure coupled solution accuracy. Modifications to the CRUD physics modeled within MAMBA include the addition of (i) dissolution kinetics to consider CRUD bursts associated with reactor power reductions, e.g. a scram, (ii) a more advanced erosion model that uses the wall shear stress in erosion kinetics calculations, (iii) the capbility to include soluble zinc as a user input within the core chemistry conditions to be considered in the species transport calculations. Additionally, the boron destruction rate model may be improved by depleting the boron within the CRUD layer directly in the nuclide depletion physics and providing the CRUD chemistry physics with a both a 
change in boron and lithium concentration.

Further investigations of the coupled physics, including when to exchange data to ensure convergence and the relative importance of the various feedback mechanisms should be carried out.

\section{Acknowledgements}

This work was supported by an NRC fellowship, the NRC faculty development grant NRC-HQ-11-G-38-0038, and the Consortium for Advanced Simulation of Light Water Reactors (www.casl. gov), an Energy Innovation Hub (http://www.energy.gov/hubs) for Modeling and Simulation of Nuclear Reactors under U.S. Department of Energy Contract No. DE-AC05-00OR22725.

\section{Bibliography}

[1] Agency, N. E. (2013). Report of the oecd/nea kaeri rod bundle cfd benchmark exercise. Technical report.

[2] Byers, W. (2000). Solubility of lithium monoborate in high temperature water. Journal of Solution Chemistry, 29(6):541-559.

[3] Castelli, R. (2010). Nuclear Corrosion Modeling. Elsevier Inc.

[4] CD-adapco (2012). STAR-CCM+Version 7.04.011 User Guide.

[5] Cho, N. Z. (2005). Fundamentals and recent ddevelopment of reactor physics methods. Technical report, Korea Advanced Institute of Science and Technology.

[6] Cohen, P. (1974). Heat and mass transfer for boiling in porous deposits with chimneys. AIChE Symposium Series, 70:138.

[7] Deshon, J. (2010). Pwr fuel crud and corrosion modeling. JOM, 63(8):64-72.

[8] EPRI (1997). Rootcause investigation of axial power offset anomaly. Technical Report 108320, EPRI, EPRI, Palo Alto, CA.

[9] EPRI (2004). Pwr axial offset anomaly (aoa) guidelines, revision 1. Technical Report 1008102, EPRI, EPRI, Palo Alto, CA.

[10] EPRI (2011). Simulated fuel crud thermal conductivity measurements under pressurized water reactor conditions. Technical Report 1022896, EPRI, EPRI, Palo Alto, CA.

[11] Gear, C. (1969). The automat integration of stiff ordinary differential equations. Information Processing, 68:187-193.

[12] Henshaw, J. (2006). A model of chemistry and thermal hydraulics in pwr fuel crud deposits. Journal of Nuclear Materials, 353:1-11. 
[13] IAEA (2007). Current trends in nuclear fuel for power reactors. Technical report, IAEA.

[14] Mathews, J. (2009). Materials and chemistry report. Technical report, EPRI, EPRI, Palo Alto, CA.

[15] Petrov, V. (2013). Impact of 3d spatial variations in fluid flow on the prediction of crud deposition in a $4 \mathrm{x} 4$ pwr sub-assembly. In NURETH-15.

[16] Station, P. V. N. G. (2004). Nuclear fuel update. Meeting with US NRC. 